\title{
Recherches en éducation
}

$26 \mid 2016$

École, citoyenneté, ethnicité

\section{Étudier l'imaginaire du stage en Institut Médico- Éducatif : un outil pour mieux situer le rapport à l'abstraction des jeunes accueillis}

Study the imagination of the intership in medical educational institute (IME): a tool to place better the report in the abstraction of the welcomed young people

\section{Antoine Agraz}

\section{OpenEdition} Journals

Édition électronique

URL : https://journals.openedition.org/ree/6625

DOI : $10.4000 /$ ree.6625

ISSN : 1954-3077

\section{Éditeur}

Université de Nantes

\section{Édition imprimée}

Date de publication : 1 juin 2016

Référence électronique

Antoine Agraz, «Étudier l'imaginaire du stage en Institut Médico-Éducatif : un outil pour mieux situer le rapport à l'abstraction des jeunes accueillis », Recherches en éducation [En ligne], 26 | 2016, mis en ligne le 01 juin 2016, consulté le 13 juin 2021. URL : http://journals.openedition.org/ree/6625 ; DOI https://doi.org/10.4000/ree.6625 


\title{
Étudier l'imaginaire du stage en Institut Médico-Éducatif : un outil pour mieux situer le rapport à l'abstraction des jeunes accueillis
}

\begin{abstract}
Antoine Agraz ${ }^{1}$

\section{Résumé}

L'objet de cet article est de décrypter l'imaginaire des jeunes accueillis en IME (Institut MédicoEducatif) du point de vue de leurs représentations de la formation en stage. Nous nous inscrivons ici dans un double ancrage théorique (Gaston Bachelard et Gilbert Durand) pour analyser la dimension symbolique de la formation en stage à l'IME, à l'aune de l'intérêt porté à cette dernière par les jeunes accueillis. Comment la formation en stage est-elle investie symboliquement par les jeunes de l'IME ? Quelles en sont les images dominantes ? L'imaginaire des jeunes de l'IME détermine leur représentation du stage, donc leur façon de s'y conduire. Nous verrons également que l'histoire des idées dans le champ du handicap intellectuel et de la pédagogie donne du sens à l'étude des nuances et des dimensions cachées liées à l'imaginaire du stage. Nous concluons en montrant comment nos résultats convoquent la question complexe et fondatrice de l'accès à l'abstraction dans le cadre du stage pour le public accueilli en IME.
\end{abstract}

\section{1. Éléments de contextualisation et position du problème}

"L'éducation des adolescents affectés d'une déficience intellectuelle accueillis en institut médico-professionnel va bien au-delà de leur initiation à des savoir-faire professionnalisants » (Horvais, 2015, p.182). Cette phrase pose le problème qui nous intéresse ici, à savoir dépasser un certain nombre de stéréotypes liés à la situation de handicap intellectuel (et à sa stigmatisation) pour parvenir à mieux cerner la face cachée, symbolique, de la formation des jeunes accueillis en Institut Médico-Éducatif (IME). Nous avons montré, dans une recherche précédente (Agraz, 2015, p.126-128), qu'une intention d'apprendre en stage était dominante dans les représentations de ce public avec handicap intellectuel, bien que cette valeur semble insuffisante pour une construction du sens des apprentissages de l'alternance. Nous avons également mis en exergue le lien entre la formation des jeunes accueillis en IME et le paradigme du «monde problématique» ( $\mathrm{p} .121)$, ce qui signifie que "les repères servant à guider l'expérience ne sauraient provenir que de l'expérience même, comme l'avait bien vu Dewey " (Fabre, 2012, p.38). C'est cette expérience que nous interrogeons ici, en nous focalisant sur l'imaginaire des stages en IME.

Nous voudrions, dans le présent article et en appui sur le cadre théorique de Bachelard prolongé par Durand, son « continuateur » (Wunenburger, 2013, p.49-52), approfondir la réflexion autour de cette intention d'apprendre et étudier comment l'image du stage accède au statut de symbole en se polarisant autour des deux régimes définis par Durand, à la suite de Bachelard : le régime diurne (rationalité scientifique) et le régime nocturne (habitation poétique du monde). L'objectif est de tenter une classification des symboles de la formation à l'IME, du point de vue des conceptions des jeunes accueillis. Comment les jeunes argumentent-ils symboliquement leur formation en stage? Quelles sont les motivations symboliques qui sous-tendent leur formation?

\footnotetext{
${ }_{1}^{1}$ Moniteur-éducateur dans un Institut Médico-Éducatif, doctorant en sciences de l'éducation au laboratoire CIVIIC de Rouen et chargé de cours à l'Université de Limoges en sciences de l'éducation et STAPS.
} 


\section{- Émergence d'un instant d'apprendre en stage}

L'instant d'apprendre s'associe à cette idée d'avoir, de la part d'un sujet, non contraint de réussir dans son action, simplement "le "loisir" de s'étonner » (Reboul, 2010, p.84). Cette posture n'en constitue pas moins une manière d'inventer de nouveaux problèmes (p.86). Le stage semble se former, dans l'esprit des jeunes de l'IME, comme instance d'étonnement. Le bien-fondé de l'étonnement réside dans le fait qu'il nous aide à prendre conscience de notre ignorance. S'étonner est au fondement du besoin d'éducation. L'éducation doit s'appuyer sur cette instance pour mobiliser l'intelligence. II faut s'étonner pour pouvoir expliquer : " C'est ainsi que l'étonnement pédagogique comporte nécessairement deux volets : l'écart, avec ce qu'on n'avait pas encore remarqué, et l'évidence qui, jusque-là, nous avait échappé » (Meirieu, 2014, p.19). Pour que l'étonnement prenne le statut de problématisation, il est nécessaire d'articuler données et conditions du problème (Fabre, 2014, p.101). Le problème se pose ici en termes d'émergence. D'ailleurs l'émergence peut être vue comme " un moment pré-intellectuel et donc un désordre » (Freiden, cité par Charbonnier, 2009, p.70). L'insignifiant a priori est porteur de signification : "Mais nous nous vouons dans ces pages à l'étude de l'insignifiant. II s'y révèle parfois d'étranges finesses. Pour les révéler, mettons-les sous le verre grossissant de l'imagination " (Bachelard, 2011b, p.109). Bachelard ajoute qu'il n'y a rien d'insignifiant dans le psychisme de l'humain (p.129). Certes cet engagement vers l'apprendre peut paraître modeste, car peu détaillé par les jeunes (Agraz, 2015, p.126). C'est une humble image.

Mais le stage n'est pas pour eux un élément anodin, ordinaire. II y a un étonnement, une rêverie derrière cet objet. La rêverie chez Bachelard est le dynamisme qui crée de l'imagination. II ne faut pas la confondre avec le rêve nocturne (Bachelard, 1997, p.36). Dans le rêve nocturne, le moi s'endort. Dans la rêverie, l'image réveille le psychisme et l'imagination est une ouverture sur un monde original. Le monde de la rêverie est sans condition : " Habitant vraiment tout le volume de son espace, l'homme de la rêverie est de toute part dans son monde, dans un dedans qui n'a pas de dehors. Ce n'est pas pour rien qu'on dit communément que le rêveur est plongé dans sa rêverie. Le monde ne lui fait plus vis-à-vis. Le moi ne s'oppose plus au monde. Dans la rêverie, il n'y a plus de non-moi. Dans la rêverie, le non n'a plus de fonction : tout est accueil » (Bachelard, 2005, p.144). La rêverie du stage constitue le premier moteur vers la connaissance objective de l'alternance à l'IME. En effet, selon Bachelard, il faut de toute façon commencer par rêver pour ensuite avoir la volonté de comprendre. L'image est première. Dès lors que l'instant présent réunit les conditions du progrès, le présent triomphe sur les autres instances du temps et c'est l'être qui s'en trouve enrichi : l'instant est tout entier dans sa dynamique de progrès, qui, ellemême, est inscrite dans un temps original. "Le temps ne dure qu'en inventant » (Bachelard, 2011a, p.86).

Nous inscrivons donc l'émergence d'un instant d'apprendre en stage à l'IME dans le modèle de la «pensée nocturne » que reprend Fabre, à la suite des travaux de Bachelard et de Durand. Fabre la définit comme une " pensée attentive aux nuances, aux contextes, à l'histoire, une pensée nocturne » (2012, p.31). Nous souhaitons donc analyser ici, à partir des représentations des jeunes en IME, les schèmes organisateurs de leur pensée du stage du point de vue symbolique. Nous nous appuierons pour ce faire sur la classification élaborée par Durand (1984). En effet, le nocturne comme structure psychique a été « repéré par Gaston Bachelard et travaillé par Gilbert Durand » (Fabre, 1995, p.180). Pour tenter d'atteindre ce «clair-obscur » du nocturne, il est nécessaire de s'intéresser au diurne (Ibid.). Nous présenterons donc, par la suite, un ensemble de données qualitatives issues de la manière dont les jeunes en IME conçoivent leur stage, en mettant ces données en rapport avec les deux régimes (diurne et nocturne) définis par Durand. 


\section{Approche théorique du clair-obscur de la formation à I'IME avec Durand}

Durand postule la « sémanticité des images » (1984, p.437). Les images ont un sens et le symbole est à la source de la pensée. L'image est première et le concept est enraciné dans un fond imaginaire. Selon Durand, l'imagination constitue la matrice de la raison : "C'est ce "sens" des métaphores, ce grand sémantisme de l'imaginaire qui est la matrice originelle à partir de laquelle toute pensée rationalisée et son cortège sémiologique se déploient» (p.27). Durand élabore trois grandes structures liées à trois rapports au monde différents : diurne, nocturne ou mystique, synthétique. Chaque structure oriente notre façon de voir le monde. Ces structures n'ont de sens qu'incarnées dans des symboles, dont nous verrons plus loin la nature du point de vue de la formation des jeunes en IME. Pour cet auteur, ce sont les représentations imagées qui sont premières, donc nous suivons Bachelard et Durand en postulant, au regard de nos résultats précédents (Agraz, 2015), que la représentation du stage comme un instant d'apprendre est première chez les jeunes de l'IME. C'est notre parti pris dans cet article.

\section{- Régime diurne de l'image}

Durand élabore une classification des structures premières de l'imaginaire selon un trajet anthropologique qui va de la réflexologie aux symboles archétypaux en deux grandes polarités : le régime diurne et le régime nocturne (Durand, 1984). Le régime diurne s'exprime par une pensée par antithèse et une logique manichéenne des images (schèmes verbaux de la séparation). Ce régime est en rapport avec les représentations des schizophrènes (p.206), où l'on repère un défaut d'unité de la pensée. Ses structures sont de type schizomorphe, en lien avec des processus d'idéalisation, de coupure, inscrites dans le registre polémique. On y trouve une obsession de la distinction et le primat de la symétrie dans la représentation. Ce régime valorise les principes d'identité, d'exclusion, de cloisonnement et de contradiction. II mobilise les schèmes verbaux de la montée (opposée à la chute) et de la distinction (p.307). Ce qui est visé par ce régime, c'est d'échapper à "l'irréversibilité du temps dans un désir d'éternité qui se manifeste à la fois par la construction de systèmes explicatifs anhistoriques et par des "gestes" héroïques. Le platonisme est - dans l'histoire des idées - la figure typique de ce régime " (Fabre, 2002, p.42).

\section{- Régime nocturne de l'image}

Le régime nocturne de l'image correspond à une "inversion des valeurs » (Durand, 1984, p.379). Les schèmes de l'intimité et de la profondeur y sont convoqués, on y rêve de bien-être, en opposition à la logique de conquête du régime diurne. Au contraire du régime diurne, il s'agit d'apprivoiser le temps, ce qui reviendrait à dire qu'il est nécessaire de maîtriser le temps de la formation en stage pour se former. Les schèmes verbaux sont ici « lier » ou « relier », « murir », "progresser ». Deux formes du régime nocturne sont définies par Durand : le nocturne mystique (antiphrasique) et synthétique (dramatique ou cyclique). Nous avons retenu, dans notre analyse du nocturne de la formation en IME, les structures synthétiques, car celles-ci se rapprochent le plus de nos résultats. Les structures synthétiques " harmonisent en un tout cohérent les contradictions les plus flagrantes " et "intègrent en une suite continue toutes les autres intentions de l'imaginaire » (p.399). Les distinctions et les oppositions sont conservées par la cohérence.

La mentalité synthétique s'inscrit dans le registre dialectique (p.403). On peut en effet affirmer une valeur mais sans pour autant déconsidérer la valeur contraire. La raison nocturne s'intéresse à la question du sens, "en essayant de comprendre comment ce sujet, cet acteur, donnent euxmêmes sens à leurs actes et quel peut-être ce sens " (Fabre, 1997, p.55). Contrairement au régime diurne, l'expérience n'est pas reproductible ici. On cherche à apprivoiser le devenir, à réconcilier les oppositions dans le temps. La recherche du compromis est constitutive du régime 
nocturne et nous verrons plus loin comment un tel compromis peut renvoyer à deux rapports à la formation différents mais non moins potentiellement complémentaires.

\section{Cadre méthodologique}

Dans une recherche précédente, nous avons examiné les représentations des jeunes de l'IME vis-à-vis du stage, en nous centrant sur le rapport au tutorat à l'aune de ce que le tuteur peut représenter sur le plan affectif pour les jeunes en stage (Agraz, 2015, p.124-126). Dans le présent article, nous avons choisi d'approfondir cette représentation tutorale, en l'examinant cette fois du point de vue de la manière dont les jeunes conçoivent la tâche à accomplir dans leur stage. Nous allons voir qu'il s'agit ici de penser le stage au travers du régime diurne de l'image, c'est-à-dire une pensée qui procède par antithèse, coupure, distinction. Notre échantillon se compose de vingt jeunes interrogés dans le cadre d'entretiens semi-directifs (garçons et filles âgés de 17 à 20 ans avec une déficience intellectuelle légère ou moyenne). Le choix d'avoir interrogé vingt jeunes s'est inscrit dans un critère de validation méthodologique lié à la dimension de saturation, entendue comme le «moment lors duquel le chercheur réalise que l'ajout de données nouvelles dans sa recherche n'occasionne pas une meilleure compréhension du phénomène étudié » (Mucchielli, 2012, p.226). Nous avons opté pour l'entretien semi-directif, outil modulable avec ce type de population, à condition de respecter certaines conditions : construction du discours, cognition, relation interviewer-interviewé (Guillemette \& Boisvert, 2003). Nous nous inscrivons dans le paradigme compréhensif où nous recherchons le sens que les jeunes de l'IME donnent au tutorat, à l'aune des significations qu'ils attribuent à la réalisation de leurs actions dans le cadre du stage. Notre analyse thématique des données s'inscrit dans une " cohérence thématique inter-entretiens" (Blanchet \& Gotman, 2001, p.98). Ajoutons que nous étudions les stages que les jeunes effectuent, à l'extérieur de l'IME, dans des domaines tels que la maçonnerie, la peinture, les activités de ménage, de cuisine et de blanchisserie ou encore les espaces verts.

\section{- Analyse des données en lien avec le régime diurne de l'image}

Sur les vingt jeunes interrogés, douze (plus d'un jeune sur deux) conçoivent le tutorat comme une aide dans la réalisation finalisée de la tâche. Parmi ces douze jeunes, nous pouvons subdiviser trois catégories :

- Cinq jeunes évoquent l'idée de finalisation de l'action avec l'aide du tuteur : «à porter des trucs lourds» (Ent $\left.\mathrm{n}^{\circ} 1^{2}\right)$, «... par exemple il fallait les sortir d'un champ (chevaux) et elle est venue m'aider pour les sortir d'un champ (Ent n8), « il m'aide à faire les silos [...] il peut m'aider à dégager les silos en côté, enlever la terre et replier la bâche dessus. II m'aide à tirer la bâche " (Ent $\left.n^{\circ} 15\right)$, "Ben c'est lui qui est responsable de toi pendant la durée du stage et qui va t'apprendre certains trucs, par exemple les trucs les plus importants quoi » (Ent n¹6), «il m’aidait à râcler les feuilles, à prendre le bac et à les mettre dedans » (Ent n¹7).

Parmi ces cinq exemples, une précision peut être apportée concernant Ent $n^{\circ} 16$ : apprendre les " trucs les plus importants " semble se rapprocher de ce qu'Astolfi nomme le « kit de survie » ou le « smic des connaissances », en faisant référence au minimum vital de savoirs que l'on confère souvent aux populations les plus démunies telles que celle accueillie en IME, alors que ce sont justement celles-ci qui auraient sans doute le plus besoin d'une exigence en termes d'apport conceptuel pour penser le monde. II apparaît que cette conception tutorale ne s'inscrit pas dans une logique d'évaluation intégrée aux apprentissages qui " caractérise le processus formatif luimême et la reconnaissance par les acteurs de leur propre évolution » (Astolfi, 2008, p.241).

\footnotetext{
${ }^{2}$ Dans un respect d'anonymat, nous avons nommé les jeunes interrogés par le sigle Ent suivi du numéro de l'entretien : Ent $n^{\circ} 1$ correspond au $1^{\text {er }}$ entretien réalisé, etc.
} 
- Cinq autres jeunes inscrivent leur représentation de l'action à réaliser à partir d'une action reproductrice par rapport au tuteur : "il te donne les instructions et puis après toi tu dois faire si tu sais bien sûr, si tu sais faire et il te dit qu'est-ce qu'il fait faire et après tu fais... » (Ent $\left.n^{\circ} 4\right)$, «Ben c'est lui qui vient pour nous aider pour faire, j'sais pas comment dire ça, pour nous dire ce qu'on a à faire, ou nous apprendre » (Ent $\left.n^{\circ} 12\right)$, «Ben des fois il me fait voir quand j'arrive pas les taies d'oreiller, il m'fait voir et après je le fais " (Ent n`13), "Il est là pour t'expliquer ce qu'il faut faire, et puis pour t'apprendre ce qu'il faut faire. Pour t'aider à faire des plats par exemple, puis pour t’aider à apprendre le ménage et tout, à faire le ménage » (Ent n`3), «Ben, à expliquer, à expliquer comment ça se passe le travail. À les écouter aussi, pour voir si tu fais quelque chose de mal ou pas bien, ça dépend » (Ent n9).

- Les deux derniers partagent une conception du tutorat centrée sur la logique productive de l'entreprise, qui ne permet pas au stagiaire d'être au premier plan en termes d'apprentissage. Le premier évoque la contrainte de temps: « ils m'ont pas beaucoup aidé parce que quand mon premier stage j'ai eu lieu, c'était, c'était à J. à T., c'était une usine alors euh... personne s'occupait de moi parce qu'y avait, ça bougeait trop vite... » (Ent $n^{\circ} 18$ ), alors que le second développe un argument lié à la qualité du produit dont les conséquences peuvent s'avérer fâcheuses si ce produit ne répond pas à la commande de l'entreprise : " cette semaine j'suis allé faire un enduit chez mon patron, il m'a expliqué à affiner l'enduit, à bien faire propre pour que ça soiye vraiment un produit vendable [...] Après y'a le nettoyage il t'explique faut faire du bon nettoyage et tout ça, parce que sinon tu vas pas être pris ou tu vas être viré euh, c'est très important le nettoyage après. La propreté du chantier et puis la propreté du produit qu'on a fait » (Ent $\left.n^{\circ} 20\right)$.

Les aspects sous-jacents à ces types de conceptions du tutorat ne sont-ils pas liés à l'idée que la réalisation doit primer sur les conditions de réalisation, et donc sur les conditions de compréhension (primat du produit de formation sur le processus de formation) ? Nos résultats paraissent converger vers un paradigme de formation pédagogiquement organisé autour du taylorisme (reproduction des tâches) et du behaviorisme (contrôle des comportements). Les schèmes correspondants sont : division, chosification, reproduction (Meirieu, 2007, p.227), ce qui se rapproche du régime diurne de l'image. Interrogeant le produit fini (le résultat) de l'élève sur le plan scolaire (comme nous tentons de le faire à partir des conceptions du tutorat centrées sur la tâche à réaliser dans le cadre du stage), Meirieu souligne bien cette distinction importante entre processus et produit (p.225). En outre, à travers la manière dont les jeunes interrogés se représentent la tâche à réaliser, il n'est pas exclu qu'ils puissent repérer, chez le tuteur de stage, des « signes extérieurs de compétence » qui peuvent leur permettre, en fonction du niveau de capacités intellectuelles, de privilégier des apprentissages moins coûteux sur le plan cognitif (cette idée émerge dans les Ent $n^{\circ} 4, n^{\circ} 13, n^{\circ} 16, n^{\circ} 18$ ).

\section{- Analyse des données en lien avec le régime nocturne de l'image}

Comme indiqué en début d'article, nous nous appuyons ici sur les données d'une recherche précédente qui a permis de mettre en relief une représentation très dominante dans l'esprit des jeunes de l'IME : l'intention d'apprendre en stage, bien que les termes de cet apprendre (ce que veut dire apprendre) ne soient pas précisés par les jeunes (Agraz, 2015, p.126-128). Notre intention est d'approfondir cette représentation de l'apprendre en la confrontant aux deux régimes de l'image définis par Durand. Au regard à la fois du caractère dominant, spontané et polysémique de cette représentation (le stage, c'est pour apprendre !), nous la classons en tant que symbole relevant du régime nocturne. Les jeunes de l'IME semblent se construire avec cette image de l'apprendre en stage qui crée du lien et le symbole de l'apprendre institue une identité commune pour ce public en situation de handicap. Comprendre la valeur symbolique des images suppose une démarche d'interprétation de ces dernières, appelée "herméneutique ». Cette discipline "privilégie l'étude des opérations intellectuelles suscitées par l'expression symbolique " (Wunenburger, 2001, p.76). Durand distingue les "herméneutiques réductives» qui réduisent les images à un «signifié sans mystère » (2008, p.43) et les "herméneutiques instauratives» qui s'intéressent à la pluralité de sens qu'elles évoquent. L'approche 
herméneutique symbolique "présuppose que l'homme se rapporte à ses images comme à des représentations à "double sens", ou dont le sens excède le donné de l'expérience" (Wunenburger, p.76).

Dès lors, il serait réducteur de cantonner le stage à son unique observation empirique. Le symbole de l'apprendre évoque d'autres possibles. Le sens de l'apprendre peut acquérir le sens symbolique et figuratif d'une possibilité d'accès à l'abstraction. Le stage devient valeur symbolique de l'apprendre dès lors qu'il se détourne de sa dimension strictement comportementaliste et productive. Cela ne signifie pas que le stage ne conserve pas une dimension comportementaliste et productive (constitutive des stages en quelque sorte), mais à celle-ci s'ajoute une nouvelle valeur : la question de l'accès aux savoirs conceptuels. En effet, le symbole nous sert à devenir différent de ce que nous sommes, à devenir autre : "La fonction symbolique est donc dans l'homme le lieu de "passage", de réunion des contraires : le symbole dans son essence et presque dans son étymologie (Sinnbild en allemand) est "unificateur de paires d'opposés" " (Durand, 2008, p.68). Le symbole est une image qui donne à vivre du sens, une quête de sens pouvant être articulée dans notre réflexion autour de la problématique de l'abstraction dans le cadre des stages en IME.

\section{Une approche archétypale de la formation à l'IME}

Les deux dimensions diurne et nocturne que nous étudions (la représentation comportementaliste de la pratique du stage et l'image dominante d'un instant d'apprendre) peuvent s'organiser en archétype car, " dans le Régime Nocturne et spécialement ses structures synthétiques, les images archétypales ou symboliques ne se suffisent plus à elles-mêmes en leur dynamisme intrinsèque, mais par un dynamisme extrinsèque se relient les unes aux autres sous forme d'un récit » (Durand, 1969, p.410). Pour éclairer notre propos, nous explorerons ce récit à partir de l'histoire des idées dans le champ du handicap intellectuel et de la pédagogie.

\section{- Imaginaire et histoire des idées}

La pensée durandienne est une pensée universelle qui mobilise une pluralité de savoirs: I'histoire des idées constitue une des disciplines à partir desquelles se construit une " science de l'imaginaire » (Durand, 1994, p.51). L'histoire des idées dans le champ du handicap intellectuel et de la pédagogie permet de donner du sens à l'étude de ces images liées à la formation des jeunes en stage, de façon à mieux comprendre ce qui soutient cette culture formative en IME. Les questions que posent les jeunes de l'IME à travers leur imaginaire de la formation se rapprochent de celles qui ont fait obstacle à la construction historique du handicap intellectuel et de la pédagogie. Nos résultats semblent concorder avec l'idée d'un archétype du stage articulé autour d'une dimension d'accès à l'abstraction, ou encore la dichotomie entre concret et abstrait. Le stage vient toucher à l'inconscient des jeunes de l'IME en apportant des images ayant un caractère collectif. Les imaginaires personnels s'enracinent dans un fond commun. L'archétype, véritable « point de jonction entre l'imaginaire et les processus rationnels » (Durand, 1984, p.63), se distingue du symbole à travers son " manque d'ambivalence, son universalité constante et son adéquation au schème» (p.63). Les schèmes sensori-moteurs mobilisés dans le travail productif réalisé par les jeunes en stage participent de la construction de cette image archétypale. Le corps tout entier collabore à la constitution de l'image. S'intéressant à l'histoire de l'éducation spécialisée à travers sa dimension anthropologique, Wacjman analyse la construction des savoirs des éducateurs à l'aune de cette dimension. Ces savoirs puisent dans la philosophie des Lumières et se fondent sur " une solide construction théorique empirique (le sensualisme) qui est comme le fil épistémologique, non pas d'une science, mais plutôt d'une discipline qui est devenue l'éducation spéciale ou spécialisée " (Wacjman, 2008, p.5). Selon cet auteur, la réalité des pratiques éducatives actuelles est fondée sur la philosophie sensualiste dont Condillac (1715-1780) s'apparente comme le chef de file. 
Wacjman situe «le rejet du caractère initial de l'idée» (p.12) comme l'un des invariants structurant l'éducation spécialisée, qu'il assimile au rejet de l'inconscient. Les méthodes médicopédagogiques actuelles concernant la déficience mentale porteraient l'empreinte de cette éducation sensualiste selon lui. Un autre auteur (Desjardins, 2011, p.183) s'inscrivant aussi dans une approche anthropologique de la déficience intellectuelle met en exergue des processus d'apprentissage de l'incompétence de la part des personnes handicapées elles-mêmes. Selon cette perspective et à l'aune de nos résultats, apprendre à être incompétent ne peut-il pas coïncider avec la manière dont les jeunes de l'IME concentrent leur attention sur le produit et non sur le processus de formation?

Meirieu reconnaît l'influence de la philosophie sensualiste chez Itard, médecin-éducateur de Victor, cet « enfant sauvage " découvert dans les bois de l'Aveyron et qu'on lui confie en 1801, à ceci près que, même s'il se réclame de Condillac, Itard, au travers de l'éducation de Victor, changera de registre ou d'« option » éducative : il réfléchira au lien entre ce que Victor est et ce qu'il veut qu'il soit. Ce lien est le lien créateur de la pédagogie. Itard se rendra compte que son projet de socialisation et d'instruction de Victor ne peut fonctionner « qu'en s'appuyant sur le donné, non un "donné définitif" mais un donné point de départ qui va être enrichi par ce qu'il permettra d'atteindre et se construira ainsi de nouvelles potentialités »(Meirieu, 1987, p.74).

Cette image archétypale du stage pourrait, selon nous, puiser également dans l'histoire des idées liées au champ de l'alternance. Les enjeux de l'alternance posent la question historique de l'articulation de deux systèmes: le système de formation et le système de production, et précisément dans la capacité du premier à se détacher du second. Meirieu y voit la tension toujours d'actualité entre deux mouvements qu'il nomme le "mouvement de didactisation » et le « mouvement de finalisation» (2009, p.289-291). Dès le XVI siècle, les compagnons du tour de France s'interrogent sur leur pratique du compagnonnage en repérant une question fondamentale : celle de la prise en compte simultanée de l'apprenti et du client. Selon Meirieu, on trouve dans les journaux des compagnons cette question de la satisfaction à la fois du client et de l'apprenti, qu'ils ont tentée de résoudre en mettant en place les cours du soir ("l'école du trait ») dans lesquels les compagnons enseignent «le trait» aux apprentis compagnons. Un siècle plus tard, il faut puiser dans Comenius (La grande didactique, $\mathrm{XVII}$ e siècle) pour retrouver cette idée et comprendre la création de l'École comme "lieu de l'erreur possible, un lieu du tâtonnement nécessaire, un lieu de l'apprentissage systématique et progressif où l'on échappe à l'aléatoire social » (p.291), lieu détaché des contraintes liées à la situation productive. Cette problématique historique du client et de l'apprenti semble se dessiner dans les propos de Ent n¹6: "Ben là pour l'instant j'apprends rien, j'donne plus un coup de main parce que ils sont après mettre une nouvelle cabine de peinture, faut défoncer le sol et tout ça, alors j'donne un coup de main. Puis j'aide les ouvriers à droite, à gauche, si ils ont besoin d'un coup de main. En ce moment, j'apprends pas grand-chose non. Non parce que j'suis plus après aider, j'suis plus après aider les autres, par exemple en carrosserie ou en mécanique, quand y'a des trucs lourds à porter, qu'on peut pas y porter tout seul, des trucs comme ça ».

\section{Discussion et conclusion}

\section{- Le problème de l'accès à l'abstraction en stage}

L'interprétation de nos résultats conduit à interroger la construction des compétences dans le cadre du stage, et plus spécifiquement à poser le problème de l'accès à l'abstraction. Peut-on soutenir ici que la question de l'abstraction soit véritablement associée à une ligne directrice ? Dans ses travaux, Horvais souligne particulièrement ce manque : "I/ serait certainement très utile de travailler à une formalisation de ces démarches pédagogiques menant à l'abstraction, en sorte qu'elles constituent un patrimoine commun reconnu, source d'inspiration pour l'ensemble des acteurs de l'éducation en IMPro» (Horvais, 2012, p.215). Nous pensons que la prise en compte de l'abstraction en stage passe par la nécessité de réfléchir au sens du mot «former ». Selon Fabre, la formation est « une action globale qui porte à la fois sur les savoirs, savoir-faire et savoir-être »(Fabre, 2006, p.22). Le processus de transformation profonde de la personne, 
inhérent à la formation et défini par Fabre, ne peut se réduire aux seuls savoir-faire et savoir-être, tel qu'une lecture partielle de notre recherche pourrait le laisser penser. S'intéresser aux savoirs construits dans le cadre du stage revient à s'intéresser à la manière dont les jeunes de l'IME y conceptualisent leur pratique. Ainsi, à partir des travaux de Vygotski sur le développement des concepts quotidiens et concepts scientifiques (1997, chap.6), nous dégageons de nos analyses précédentes des éléments en relation avec ces travaux. Les concepts quotidiens font appel à des croyances relevant d'un vécu quotidien (Saussez \& Paquay, 2004, p.5) alors que les concepts scientifiques se rapportent à des "savoirs constitués" (p.17). Nos résultats en rapport avec le régime diurne de la formation (pratique comportementaliste, réussite immédiate de l'action) semblent coïncider avec la définition des concepts quotidiens en termes de croyances ancrées dans le vécu quotidien du stage. De la même manière, nos résultats en rapport avec le régime nocturne (intention d'apprendre, sens symbolique de l'apprendre) semblent concorder avec la définition des concepts scientifiques en ce que ces résultats autorisent une possibilité d'accès à un degré de généralité plus important (conceptualisation plus grande) de façon à mieux résoudre les problèmes qui se posent en stage. Cette tension entre concepts quotidiens et concepts scientifiques n'est pas spécifique au public des IME puisqu'elle concerne également les stages dans les métiers d'enseignement : "Or, c'est souvent le dialogue entre les concepts quotidiens et les concepts scientifiques qui posent problème aux stagiaires » (Ibid.). Toutefois, les difficultés inhérentes à la population étudiée ici (handicap intellectuel) constituent, en quelque sorte, un effet de loupe dans ce domaine.

\section{- Tension entre « temps vécu » et «temps pensé » du stage}

Comme nous l'avons indiqué ci-avant, réduire la formation en stage aux savoir-faire et savoirêtre ne permet pas de prendre en compte la dimension symbolique émanant des représentations des jeunes de l'IME. Nous avons vu que Bachelard et Durand soulignaient l'importance d'associer à l'imagination un dynamisme de rupture. L'intérêt de notre contribution est justement de mettre en lumière cette dimension symbolique, cet imaginaire de la formation qui se rapproche d'un temps pensé du stage, en tension avec un temps vécu. Selon Bachelard, le temps pensé est lié « aux engagements de la pensée rationnelle » (Vinti, 2008, p.190). Ce temps de la connaissance est fortement attaché à l'Etre : "C'est dans ce temps qu'un fait devient un facteur. On qualifie mal ce temps en disant qu'il est abstrait, car c'est dans ce temps que la pensée agit et prépare les concrétisations de l'Etre » (Bachelard, 2001, p.17). Le temps vécu du stage, quant à lui, correspond au "temps biologique, au temps perçu immédiatement » (Vinti, 2008, p.191), c'est-à-dire, d'après nos analyses, au temps du stage qui consiste à mobiliser son corps dans une perspective de finalisation de la tâche (cf. partie Analyse des données en lien avec le régime diurne de l'image). À travers nos résultats, nous pouvons nous demander dans quelle mesure l'imaginaire du stage peut constituer la voie qui conduit à la dimension rationnelle de la formation. Quel dialogue potentiel peut-il exister entre le jeune de l'IME et le monde de l'abstraction (accès aux savoirs conceptuels) ? Les conditions de possibilité du passage de l'instant d'apprendre vers le processus «apprendre" (Houssaye, 2009, p.18-20) semblent inscrites dans l'enrichissement de l'expérience au niveau intellectuel. L'intention d'apprendre en stage exprimée par les jeunes de l'IME fait appel au processus par lequel le jeune accède au savoir. C'est ce processus qui a valeur symbolique et se trouve en quelque sorte dissimulé derrière une pratique comportementaliste du stage. Dès lors, un accès à la dimension rationnelle de la formation passe par une meilleure prise de conscience des concepts scientifiques du stage, dont la caractéristique est la capacité de « définition verbale initiale " (Vygotski, 1997, p.274). Cette capacité est réellement déficiente chez le public accueilli en IME: nos résultats d'observation antérieurs peuvent en témoigner (Agraz, 2015, p.126). Ajoutons que cette difficulté est à la fois nécessairement dépendante du « processus de formation » et « des exigences de la profession (et de l'exercice professionnel) en matière d'utilisation de connaissances scientifiques" (Saussez \& Paquay, 2004, p.5). Dans ces conditions, il paraît donc essentiel qu'un étayage pédagogique puisse intervenir au niveau du "dire sur le faire ", de façon à ouvrir l'avenir de la formation en stage des jeunes accueillis en IME (Feuilladieu, Gombert, Assude, 2015, p.8). Nos résultats convergent donc vers l'idée que les jeunes de l'IME dirigent leur attention vers les savoirs pragmatiques du stage. En se focalisant sur la finalisation de la tâche, ils ne sont pas conduits à donner le maximum sur le plan cognitif et ne sont pas préparés à une 
autoévaluation d'eux-mêmes. Pourtant, les travaux de Vygotski (Barisnikov \& Petitpierre, 1994, p.170) ont bien montré que le développement des capacités d'abstraction pour un public avec déficience mentale est un enjeu d'importance. D'autres auteurs s'inscrivent dans cette même perspective, à l'instar de Mayen (2000) ou Horvais (2012). Les progrès en termes d'apprentissage passe par la capacité à articuler savoir empirique et savoir conceptuel.

\section{Conclusion}

Notre objectif était de mieux comprendre le champ imaginaire de la formation à l'IME du point de vue des représentations des jeunes accueillis dans ce type de structure spécialisée. Pour ce faire, nous avons interrogé le passage entre le stage tel qu'il est conçu par les jeunes de l'IME (à travers l'étude des schèmes diurnes qui le cantonnent à la réussite immédiate de l'action concrète) et une perspective nocturne d'accès à l'abstraction, à des savoirs conceptuels soulevés par l'image dominante d'un instant d'apprendre, plus encline aux enjeux d'une alternance (recherche de schèmes alternatifs). Dans la pensée nocturne, ces deux moments temporels sont à appréhender dans leur singularité respective et dans leur rapport dialectique. Et bien qu'il convienne de ne pas basculer dans une facilité d'esprit consistant à les mettre sur le même plan (Bachelard, 2007, p.19), le schème diurne de la réussite immédiate de l'action dans le cadre du stage semble phagocyter l'image nocturne, de sorte qu' " au lieu de nous faire rêver et parler, elle nous fait agir » (Bachelard, 2014, p.6), en intériorisant les traits de la perception présente (finalisation de la tâche productive). L'image de l'apprendre est certes première dans les représentations des jeunes, mais leur façon de concevoir la pratique du stage vient en quelque sorte obérer cette image initiale en la traduisant par des schèmes articulés autour d'un ordre empirique du stage. Cette double base (diurne-nocturne) apparaît comme la condition de possibilité d'une "anthropologie du très proche » (Gardou, 2013), entendue comme le fait de s'intéresser au vécu des personnes en situation de handicap et au décryptage du sens de ce vécu. D'où l'importance, avec Bachelard et Durand, d'associer à l'imagination un dynamisme de rupture. Pour éviter de basculer dans un immobilisme de l'esprit, le pédagogue doit s'inscrire dans une pédagogie du risque et "faire rêver l'intelligence" (Jean, 1983, p.123-124) de l'apprenant, de manière à transformer les conditions de possibilité du stage.

La philosophie de l'instant de Bachelard, que nous avons mobilisée pour penser le stage en IME, est intéressante en ce qu'elle permet une réflexion sur la capacité potentielle des jeunes à dire «Je », à être sujet de leur formation. L'unité du moi passe par la réunion de toutes les expériences vécues par le jeune dans son stage. Dans ce contexte, peut-on avancer que le jeune de l'IME a véritablement conscience de sa formation en tant que telle ? La tâche du pédagogue s'inscrit sans doute dans cet étayage à la prise de conscience de ce que signifie l'alternance et l'apprendre à l'IME, de façon à aider le jeune à pouvoir véritablement dire « je », c'est-à-dire être en mesure d'assumer la continuité de son expérience, l'aider à être le plus responsable possible des changements pouvant intervenir dans son parcours de formation, autant d'éléments favorisant un meilleur accès à la dimension rationnelle de la formation. Toutefois, ce que cette recherche ouvre comme questionnement transversal à l'aune de nos résultats, c'est la question de l'accès au patrimoine commun de la formation (Gardou, 2012), dont les jeunes accueillis en IME semblent quelque peu dépossédés. À l'inverse de ce que prône Gardou en se situant dans une approche inclusive (dans laquelle le sujet doit avoir la possibilité de "se produire»), il semble que nous soyons davantage ici sur le terrain du manque ((Feuilladieu, Gombert, Assude, 2015, p.8).

\section{Bibliographie}

AGRAZ A. (2015), « Le rapport à la formation des jeunes accueillis en IME : une tension entre hétéroformation et autoformation? ", La nouvelle revue de l'adaptation et de la scolarisation, n69, p.119-130.

BACHELARD G. (1997), La psychanalyse du feu, Saint-Amand, Gallimard. 
BACHELARD G. (2001), La dialectique de la durée, Paris, Presses Universitaires de France, $3^{\text {ème }}$ édition "Quadrige ».

BACHELARD G. (2005), La poétique de la rêverie, Paris, Presses Universitaires de France, $6^{\text {ème }}$ édition "Quadrige ».

BACHELARD G. (2007), Le matérialisme rationnel, Paris, Presses Universitaires de France, $3^{\text {ème }}$ édition «Quadrige».

BACHELARD G. (2011 a), L'intuition de l'instant, Paris, Presses Universitaires de France, $7^{\text {ème }}$ édition "Quadrige ».

BACHELARD G. (2011 b), La poétique de l'espace, Paris, Presses Universitaires de France, $10^{\text {ème }}$ édition «Quadrige ».

BACHELARD G. (2014), L'Air et les songes. Essai sur l'imagination du mouvement, Paris, Presses Universitaires de France, $10^{\text {ème }}$ édition.

BARISNIKOV K. \& PETITPIERRE G. (dir.) (1994), Défectologie et déficience mentale. Vygotsky, Paris, Delachaux et Niestlé.

BLANCHET A. \& GOTMAN A. (2001), L'enquête et ses méthodes : l'entretien, Paris, Nathan.

CHARBONNIER S. (2009), Deleuze pédagogue. La fonction transcendantale de l'apprentissage et du problème, Paris, L'Harmattan.

DESJARDINS M. (2011), « La construction anthropologique des problèmes sociaux. L'exemple de la déficience intellectuelle ", Problèmes sociaux. Tome 1, Théories et méthodologies, H. Dorvil \& R. Mayer (dir.), Québec, Presses de l'Université du Québec, p.175-190.

DURAND G. (1984), Les structures anthropologiques de l'imaginaire, Paris, Dunod, 10 ème édition.

DURAND G. (1994), L’imaginaire. Essai sur les sciences et la philosophie de l'image, Paris, Hatier.

DURAND G. (2008), L'imagination symbolique, Paris, Presses Universitaires de France, $5^{\text {ème }}$ édition "Quadrige ».

FABRE M. (1995), «La formation comme régime nocturne: raison narrative et formation », Education Permanente, $\mathrm{n}^{\circ} 122$, p.179-189.

FABRE M. (1997), «La formation inconcevable et pourtant pensable. De quelques dualismes en formation », Penser l'éducation, vol. 1997-2, p.53-63.

FABRE M. (2006), Penser la formation, Paris, Presses Universitaires de France, $2^{\text {ème }}$ édition.

FABRE M. (2012), «Éloge de la pensée nocturne », Histoires de nuits au cours de la vie, C. Schmutz-Brun (coord.), Paris, L'Harmattan, p.59-67.

FABRE M. (2014), « De l'étonnement au problème », Education Permanente, n²00, p.97-105.

FEUILLADIEU S., GOMBERT A. \& ASSUDE T. (2015), « Vers l'accessibilité aux savoirs des élèves en situation de handicap », Recherches en Education, n²3, p.3-10.

GARDOU C. (2015), La société inclusive, parlons-en! II n'y a pas de vie minuscule, Toulouse, Erès, $1^{\text {ère }}$ édition 2012.

GARDOU C. (2013), « Une anthropologie du très proche », Vers une anthropologie du handicap, R. Grim (dir.), Paris, L'Harmattan, p.59-67.

GUILLEMETTE F. \& BOISVERT D. (2013), «L'entrevue de recherche qualitative avec des adultes présentant une déficience intellectuelle », Recherches qualitatives, vol. 23, p.15-26. 
HORVAIS J. (2012), Qu'apprend-on en IMPro ? Les apprentissages proposés aux adolescents déficients intellectuels dans IMPro: quels choix, quelles pratiques, pour quoi faire?, Thèse de doctorat en Sciences de l'éducation sous la direction du professeur Charles Gardou, Université Lumière Lyon 2.

HORVAIS J. (2015), « Apprendre à contribuer au bien commun : essai sur les déclinaisons inclusives de l'action éducative ", La nouvelle revue de l'adaptation et de la scolarisation, n69, p.181-190.

HOUSSAYE J. (2009), "Le triangle pédagogique ou comment comprendre la situation pédagogique », La pédagogie : une encyclopédie pour aujourd'hui, J. Houssaye (dir.), Issy-Les-Moulineaux, ESF, 8 ème édition, p.1324.

JEAN G. (1983), Bachelard. L'enfance et la pédagogie, Paris, Scarabée.

MEIRIEU P. (2007), La pédagogie entre le dire et le faire, Paris, ESF, $3^{\text {ème }}$ édition.

MEIRIEU P. (2009), “ Objectif, obstacle et situations d'apprentissage », La pédagogie : une encyclopédie pour aujourd'hui, J. Houssaye (dir.), Issy-Les-Moulineaux, ESF, $8{ }^{\text {ème }}$ édition, p.289-299.

MEIRIEU P. (2014), « Mais où est donc passé l'étonnement ? », Education Permanente, n²00, p.17-21.

MUCCHIELLI A. (dir.) (2012), Dictionnaire des méthodes qualitatives en sciences humaines, Paris, Armand Colin, $3^{\text {ème }}$ édition.

REBOUL O. (2010), Qu'est-ce qu'apprendre ? Paris, Presses Universitaires de France.

SAUSSEZ F. \& PAQUAY L. (2004), "Tirer profit de la tension entre concepts quotidiens et concepts scientifiques. Quels espaces de formation et de recherche construire? », Entre sens commun et sciences humaines, De Boeck, p.115-138.

VINTI C. (2008), «Sur le sujet: Bachelard contre Bergson », Bachelard et Bergson. Continuité et discontinuité ? F. Worms \& J.-J. Wunenburger (dir.), Paris, Presses Universitaires de France, p.183-195.

VYGOTSKI L. (1997), «Étude du développement des concepts scientifiques pendant l'enfance », Pensée \& langage, Paris, La Dispute, p.271-413.

WACJMAN C. (2008), Les fondements de l'éducation spécialisée. Principes, méthodes, enjeux, Paris, Dunod.

WUNENBURGER J.-J. (2001), Philosophie des images, Paris, Presses Universitaires de France, $2^{\text {ème }}$ édition.

WUNEnBuRgeR J.-J. (2013), L'imaginaire, Paris, Presses Universitaires de France, «Que sais-je », $2^{\text {ème }}$ édition. 